\title{
DIEZ CANCIONCITAS POPULARES EN UN MANUSCRITO VALENCIANO DEL SIGLO XVI
}

Ya está visto que nunca podremos ponerle punto final a nuestro conocimiento de las viejas canciones populares que se cantaban en la España del siglo xvi. Recientemente he podido consultar un manuscrito del que no tenía noticia, el cual, entre otras poesías, contiene nuevas versiones de ocho cantares incluidos en mi Corpus de la antigua lírica popular ${ }^{1}$, y dos no recogidos en él.

Se trata del Tiple y el Altus de un cancionero polifónico ${ }^{2}$ recopilado, al parecer, en Valencia, calculo que entre 1560 y 1582. A Valencia remiten, conjuntamente, la lengua de algunas composiciones, la presencia de varias poesías que también se encuentran en recopilaciones valencianas y la inclusión de un Miserere atribuido al compositor Ginés Pérez (o Juan Ginés Pérez), que

1 Corpus de la antigua lírica popular hispánica (siglos xo a xvii), Castalia, Madrid, 1987; $2^{\text {a }}$ ed., 1990. No repetiré los datos bibliográficos ahí registrados.

${ }^{2}$ El códice, que está sin clasificar, se encuentra en la Sección de Música de la Biblioteca Nacional de Madrid. Debo su conocimiento a mi amigo Luis Robledo, quien a su vez lo conoció gracias al bibliotecario José Carlos Gonzálvez. Los dos pequeños cuadernos apaisados están encuadernados en pergamino, y sobre él se lee, respectivamente, Tiple y Altus (abreviaré $T:$ y $A$ ). No hay ningún otro título ni encabezado. Los bordes parecen de encaje, pues el papel está severamente dañado por la polilla. El Tiple suele ofrecer versiones mejores. En el Altus han desaparecido casi todos los números de los folios, que he suplido sólo cuando ello no significaba hojear demasiado el códice. La disposición de las composiciones varía en las dos partes, y por fortuna lo que no se lee claramente en una de ellas puede completarse con la otra. Preparando este trabajo, me encuentro una mención del manuscrito en el artículo de J. Romeu Figueras aludido abajo, nota 8; menciona, p. 101, "un manuscrito musical existente en Madrid, Biblioteca Nacional, sección de Música, sin signatura, que yo sepa"'; la nota 7 dice: "Sólo se han conservado dos cuadernos, en muy mal estado, de pequeño formato y apaisados, correpondientes al altus y tiple; último tercio del siglo xvı. Debo el conocimiento de este manuscrito a mi buen amigo don Jaime Moll Roqueta". 
fue maestro de capilla en la Catedral de Valencia ${ }^{3}$. En cuanto $a$ la fecha: la mayoría de los estribillos de cantares que aquí comen. taré se encuentra en recopilaciones de los años 1560-1576: los que he numerado 2 y 5 figuran en las Obras póstumas de Férnandez de Heredia (Valencia, 1562); el 4 (y otros dos citados en la notí 7) en la Flor de enamorados (primera edición conocida, Barcelona 1562); el estribillo del núm. 3, en el Villete de amor de Timoned ([Valencia], s.f.); los de los núms. 1, 3, 4 (más tres citados en lá nota 7) están en el manuscrito Cancionero sevillano de la Hispanic So ciety (ca. 1568) ${ }^{4}$; el del núm. 3 , en el manuscrito 17.698 de lic B.N.M., al que he llamado Cancionero toledano (1560-1570); el de 1 , en un pliego suelto de 1570 . Otra versión del villancico núm 7 figura en la Recopilación de sonetos y villancicos de Juan Vásquez (Sevilla, 1560) y otra del núm. 8, en el Libro de mosica en cifras parc vihuela intitulado el Parnasso de Esteban Daza ([Valladolid], 1576) Un dato más lo constituyen las fechas en que Ginés Pérez fue maes. tro de capilla en Valencia: entre 1581 y 1595 (ver nota 3). Más adelante explicaré por qué señalo el año de 1582 como fecha tope

Aparte de varias piezas litúrgicas, con texto latino, y de la en salada "Oýd, oýd, los viuentes [sic]" (La justa), de Mateo Fleche el Viejo ${ }^{5}$, la colección comprende la letra de diez y nueve villan cicos profanos, en varios de los estilos habituales en la segunde mitad del siglo XvI, desde el cortesano ${ }^{6}$ hasta el popular-tradicio

${ }^{3}$ Ver New Grove Dictionary of Music and Musicians, 1980, t. 14, p. 368; y José Climent Barber y Joaquín Piedra Miralles, Juan Bautista Comes y su tiem po. Estudio biográfico, Comisaría Nacional de la Música, Madrid, 1977, pp 67-68.

${ }^{4}$ Además, varias mencionadas abajo, notas 6 y 7: "Quién te hizo Juan...", “Torna, Mingo, a enamorarte...", "'Zagala como (más que) la: flores...". En la edición del Cancionero sevillano que preparamos José J. La brador Herraiz, Ralph DiFranco y yo podrán verse documentadas esas poesías

${ }^{5}$ Como reveló José Romeu Figueras, este compositor catalán estuvo re lacionado, en la cuarta década del siglo XvI, con la corte valenciana de dor Fernando de Aragón ("Mateo Flecha el Viejo, la corte literariomusical del du que de Calabria y el Cancionero llamado de Upsala", $A n M, 13,1958,25-101$ en especial, pp. 32-34). Sus ensaladas circulaban todavía en las útimas déca das del siglo XVI, como lo prueban, además del nuestro, los dos manuscrito: barceloneses y la edición de Praga, 1581 (cf. Mateo Flecha, Las ensaladas, ed Higinio Anglés, Diputación Provincial de Barcelona-Biblioteca Central, Bar celona, 1954, pp. 33-35).

6 "Justa cosa fue quereros, / no ai maior bien que miraros..." ( $T 37 \mathrm{v}$ 38v; de Garci Sánchez de Badajoz; tal como figura en su "Despedido de con suelo...", Cancionero general, 1514); “Puy fortuna m'a llevat / lo major bé qu 
nal, pasando por el pastoril y popularizante ${ }^{7}$.

Daré cuenta de los textos de tipo popular; los he numerado para fines de este trabajo. De los números 1 y 2 conocíamos ya el estribillo y la glosa (culta). De los núms. 3 a 6 sólo nos era conocido el estribillo, no la glosa, la cual en 3 y 4 es culta y en 5 y 6 , de tipo popular. El villancico entero - estribillo y glosade los números 7 y 8 , popular y sin duda antiguo, se había encontrado en una sola versión; es importante haber dado con otra, que además difiere en detalles significativos. Finalmente, los núms. 9 y 10 son canciones que no recuerdo haber visto antes.

\section{VilLANGicos CON ESTRIBILLO DE TIPO POPULAR Y GLOSA CULTA}

[1] Véante mis ojos y muérame yo luego, dulce amor mío y lo que yo más quiero. $(A[10] \mathrm{v} ; T[14] \mathrm{r})$.

Va seguido de una estrofa ("A trueque de verte, / la muerte m'es vida...'’), que es la primera de la glosa de Jorge de Montemayor. Como puede verse en Corpus, 428, el villancico de Montemayor figura también en otro cancionero manuscrito ligado a Valencia, el 2621 de la B.N.M. (segundo tercio del siglo xvI), en el Cancionero sevillano y en un pliego suelto de Granada, 1570. El estribillo fue glosado además por Andrade Caminha y reproduci-

tenia..." ( $T$ 44r-45r); "No seas cruel, señora, / yo te auiso / que crueldat no reyna'n paraíso..." (T 8r).

7 "Mi ganado busque dueño..." ( $T$ 50r-51r); "Quién te hizo, Juan Pastor. . " (T 51v-52v; también es el Sarao de amor de Timoneda, 1561, y Cancionero sevillano); "Torna, Mingo, a enamorarte..." (T45v-46v; también en Cancionero sevillano; cf. el "Vuelve, Gil, a enamorarte", de la Flor de enamorados); "Olvida, Bras, a Constanza" (sólo copiado ese verso, T 60r-61v). Villancico pastoril es igualmente el reproducido aquí con el número 10. Otras semipopulares: "Zagala como las flores..." ( $T$ 47r-48r; en Flor de enamorados y en Cancionero sevillano); "Ai, bona moreta, / qui us ama sinó io? / Gratioseta, gratioseta, / seruidor vostre só. // Recor-vos, señora / del bon amllimen [sic], / que fes en vostra casa / lo primer parlament $[A$ : parclamem]; / girau-me la cara, / ai deo, y què's azò? / De mi us os andita, / quaque fals amó. / Gratioseta. .." ( $T$ y $A$ 63v-64v); y un improvisado cantar a un misacantano: "Galán que canta la misa / yjo es de uilla de lo principal, / y oy le cantan todos la gala, / pues á llegado al divino altar [decía: "pues el pelejo dexó por la fe"']. // Oy aquesta noble uilla / festeja su pastor santo, / y ansí $[A$ : ahi $]$ con grande alegría / siruen a Bartolomé" ( $T$ 68v-69r, $A$ [14]v-[15]r). 
do por Lo Frasso. La conocida versión a lo divino "Véante mis ojos, / dulce Jesús bueno... "' (Corpus, 1385), tradicionalmente atribuida a Santa Teresa, se cantaba todavía en el siglo Xvir y parece haber seguido viva en la tradición oral.

[2] Pux que no.m uoleu amar, no me n'é cura, que a mi non fallirà bona uentura.

$$
\text { (A 40r-41v; T 39r-40v). }
$$

A este estribillo (Corpus, 675 A) le sigue una glosa que es, con algunas variantes, la que figura en las Las obras de Fernández de Heredia (Valencia, 1562); dice así en el cancionero: "Ja, per a fer que.n vullau, / é fet tot lo que [he] pogut, / y uech que menis só uolgut, / que [.n] mili coses o mostrau. / Y pux tan nos declarau, / no me n'é cura, / que a mi non fallirá / bona uentura'.

José Romeu Figueras publicó en 1969 un detenido estudio sobre las varias y variadas versiones de este cantar ${ }^{3}$. El estribillo se cantaba - y bailaba-, de otra manera, en Mallorca en 1514, seguido de dos estrofas paralelas (Corpus, $675 \mathrm{~B}$ ), al estilo popular. En castellano, con más variantes y otra glosa paralelística (Corpus, $674 \mathrm{~B}$ ), figura en el extraordinario pliego suelto Cantares de diuersas sonadas. . . ansi para baylar como para tañer, que tuve ocasión de prologar en 1952 y que en 1969 Romeu consideraba "impreso en Valencia, probablemente entre 1530 y 1535 '”. Dice así el estribillo castellano: "Pues no me queréys amar / como soléys, / si de otra me enamorare, / no me culpéys". Finalmente, este estribillo castellano ("Pues no me queréys hablar... ") figura también (Corpus, 674 A) en otras tres fuentes, dos de ellas musicales: con glosa culta, en el llamado "Cancionero Musical de Barcelona" (Biblioteca de Cataluña, ms. M 454), asimismo relacionado con la ciudad de Valencia ${ }^{10}$, y en el Libro de cifra nueva para tecla, harpa y vihuela de Venegas de Henestrosa (1557), donde sólo se copia el primer verso; la tercera es una ensalada contenida en un

${ }^{8}$ Se trata del artículo "La colección "Cantares de diversas sonadas" y la serie «Pus que no.m voleu amar» - «Pues (que) no me queréys amar» $\mathrm{O}$ «hablar"', AnM, 22 (1969), 97-143, en especial, pp. 100-105, 109-110; ROMEU reproduce, p. 101, los cuatro versos finales de la versión incluida en nuestro cancionero.

${ }^{9}$ Ibid., p. 141.

${ }^{10}$ Romeu Figueras, “Mateo Flecha el Viejo...”, pp. 35 ss. 
pliego suelto de la British Library ${ }^{11}$. De todos estos testimonios, el de nuestro cancionero parece ser el más tardío; esta canción, que tanta fortuna alcanzó por la cuarta década del siglo, seguía viva cuarenta años después; habría que ver si existe relación entre sus tres versiones musicales.

[3] Morenica, no desprecies la color morena, que aquesa es la color buena

$$
\text { (A 42r-43r; } T \text { 41r-42v). }
$$

No conocíamos la estrofa glosadora: "El mesmo que nos crió / mirad si sabría escoger: / de hermosa quiso nacer / y morena la escogió. / No's tengáis en poco, no, / por ser morena, / que aquesa es la color buena'. El estribillo (Corpus, 145 B), variado y seguido de sendas glosas diferentes, aparece también en el Cancionero sevillano, el Cancionero toledano y el Villete de amor de Juan Timoneda. Otro manuscrito del siglo xvi nos presenta lo que puede haber sido una versión más antigua: "Morenica, no desprecies la color, / que la tuya es la mejor" (Corpus, 145 A).

[4] Váseme mi amor, quiéreme dexar: aunque soi morena, no soi d'olvidar. ( $T$ 48v-49v; $A$ 49r-50r).

La glosa consta de dos estrofas, la primera de las cuales reza: “ ¡Ai, qué d'esperanças / que arebata el viento! / Ai, qué de mudanzas / hace vn pensamiento! / $\mathrm{iAi}$, que no ai contento / que pueda durar! / Aunque soi morena, / no soi de olvidar". También esta glosa difiere de las hasta ahora conocidas (ver Corpus, 530): la del Cancionero sevillano y la de la Flor de enamorados, famoso cancionero que, como propone $\mathrm{Romeu}^{12}$, fue recopilado por $\mathrm{Ti}$ moneda y originalmente publicado en Valencia, posiblemente en 1556. En 1625 incluyó Gonzalo Correas el estribillo entre las seguidillas viejas citadas en su Arte de la lengua española castellana. Estas tres fuenies presentan el cantarcillo de manera ligeramente di-

${ }^{11}$ Chiste nueuo co seys Romances... por francisco de Arguello. . Puede verse ahora en Pliegos poéticos españoles de la British Library, Londres, ed. facs. con estudio de Arthur Lee Francis Askins, Joyas Bibliográficas, Madrid, 1989, núm. 3.

12 Joser Romeu i Figueras, Joan Timoneda i la "Flor de enamorados", cançoner bilingüe. Un estudi $i$ una aportació bibliogràfica, Barcelona, 1972. 
ferente de la nueva versión encontrada, pues en ellas comienza: "Vanse mis amores, / quiérenme dexar..."

\section{VILLANCICOS CON ESTRIBILLO POPULAR CONOCIDO} Y GLOSA POPULAR DESCONOCIDA

En dos casos nos encontramos con estribillos populares que constan en otras fuentes antiguas, pero sin la glosa de tipo popular que los acompaña en el cancionerillo valenciano. Como no son muchas las glosas populares conservadas, estos dos testimonios resultan especialmente valiosos.

[5] Quien a dos amores ama a traición le saquen el alma.

Quien a dos amigas ama de dentro de aquesta villa a traición le saquen el alma delante la más garrida. $(A 43 \mathrm{v}-44 \mathrm{v} ; T 42 \mathrm{v}-43 \mathrm{v}, \mathrm{v} .4$ ante)

El estribillo (ver Corpus, 2024) apareció glosado en estilo culto en la Farça hecha a manera de visita de Juan Fernández de Heredia, y tiempo después en una ensalada del segoviano Antonio Balvas Barona; el Comendador Griego lo citó entre sus refranes, con la variante "le sacan el alma". Por el testimonio de Fernández de Heredia sabíamos que este proverbio se cantaba y por el de Balvas, que también se bailaba ("al son de su vayle cantan"); ahora lo tenemos ya con música y con una glosa bailadera. Hecha según una conocida técnica tradicional ${ }^{13}$, la estrofa glosadora puede haberse duplicado paralelísticamente (como en Corpus, 67): “... de dentro de esta bailía... / delante la más lozana”.

[6] Vente a mí, torillo, torillo fosquillo, torillexo, y uente a mí.

13 La que he llamado "despliegue"' (cf. "Glosas de tipo popular en la antigua lírica", $N R F H, 12,1958,301-334$, y Estudios sobre lírica antigua, Castalia, Madrid, 1978, pp. 267-308). Cf. Eugenio Asensio, Poética y realidad en el cancionero peninsular de la Edad Media, $2^{\text {a }}$ ed., Gredos, Madrid, 1970, pp. 200-203. Para el verso "de dentro de aquesta villa", véase Corpus, 67, 1537 B. 
En la plaza de San Pedro

cor[r]ían vn toro negro;

salió la niña en cabello:

- ¡A mí, a mí, que no al cauallero!

¡Torillexo, y vente a mí!

Vente a mí, torillo, torillo fosquillo, torillexo, y uente a mí.

$$
\text { (T 62r-63r; } A \text { 62r-63r). }
$$

Sólo conocíamos hasta ahora el estribillo, en varias versiones, todas ligeramente diferentes de ésta y entre sí y todas recogidas en fuentes de muy de fines del siglo XVI y del XviI(ver Corpus, 2178 A, B, G). Es probable que nuestro texto sea el testimonio más antiguo. La versión más parecida a la nuestra aparece como estribillo de un poemita chusco, sobre marido cornudo, que Pedro de Flores publicó en el Ramillete de Flores. Cuarta parte de Flor de romances (1593) y en la Sexta parte de flor de romances nuevos (1594); comienza así "Estando vn día en la villa, / porque le regozijasse, / me mandó que le cantasse / mi marido vna coplilla; / por quitarme de renzilla, / vcho ho, le respondí, / vente a mí, torillo fosquillo, / toro fosco, y vente a $m \imath$ ". Luego Covarrubias recogerá el mismo "cantarcillo antiguo" en su Tesoro, en versión también muy parecida: "Vente a mí, torillo hosquillo, / torillejo, vente a mí" (Corpus, 2178 A). El cantar sería muy del gusto de los autores de bailes dramáticos, entremeses y mojigangas del siglo XVII.

En las varias utilizaciones del cantar el toro simboliza cosas diferentes, entre ellas, al amor, y se diría que el "toro negro" que aparece en la glosa de nuestro cancionero es precisamente ese "torillo de amor" que encontramos en una composición del xvir (ver Corpus, 2179, nota). Por su tema y por su forma es muy curiosa esa glosa de tipo popular que sigue al estribillo; no tiene, que yo recuerde, un paralelo en el repertorio conocido; desafiando directamente al toro negro, la niña en cabello desplaza al caballero que pretendía torearlo.

VILLANCICOS CON ESTRIBILLO Y GLOSA POPULARES CONOCIDOS EN UNA SOLA VERSIÓN, CON VARIANTES DE PESO

[7] Si el pastorcico es nueuo y anda enamorado, 
si d'amor adolesce, perdido es el ganado.

-Dime, pastorcico del cuerpo lozano, ¿cúias son las vacas que andan por el vado?

-Que vuestras son, señora, y mío es el cuidado.

Si d'amor adolesce, perdido es el ganado.

( $T 36 \mathrm{r}-37 \mathrm{r} ; A$ s.f., v. 10 omite $e s$ ).

La única versión hasta ahora conocida (Corpus, 1155) está en el segundo cancionero polifónico de Juan Vásquez, su Recopilación de sonetos y villancicos (Sevilla, 1560). En el cancionero valenciano la música es visiblemente distinta de la maravillosa composición del extremeño y el poema presenta divergencias textuales. Los versos 3-4 dicen en Vásquez "si se descuyda y duerme, / ¿quién guardará el ganado?'?. La glosa expresa básicamente lo mismo en las dos fuentes, pero con distinta asonancia: $i-o$ en Juan Vásquez, $a$-o en nuestra nueva versión, como si se tratara de dos estrofas paralelas que fueron a dar a lugares distintos. El final de la nueva estrofa recuerda el de la canción extremeña citada en la nota del Corpus: "-Son de usté, señora, / y el cuidado es mío"; quizá esta última fuera la forma popular de la estrofa de Juan Vásquez, acaso retocada por él' ${ }^{14}$.

[8] Gritos daua la morenica sola en l'oliuar, que los ramos haze temblar.

Morenica cuerpo garrido (bis) gritos daua so el uerde oliuo, sola en l'oliuar que los ramos haze temblar.

${ }^{14}$ Cf: en M. Frenk, "Sobre los textos poéticos en Juan Vásquez, Mudarra y Narváez", Estudios sobre lírica antigua, pp. 199-200, y "Apostillas a un artículo sobre el romancero", $N R F H, 12$ (1958), 58-60. Tendía yo antes a considerar la glosa como "fabricada" por Juan Vásquez sobre elementos tradicionales (entre líricos y romancescos), pero la nueva versión apunta más bien a la "autenticidad folklórica" de la estrofa, salvo quizá el " $m i$ señora" y el "suspiro". 
Gritos daua la morenica

sola en l'oliuar,

que los ramos haze temblar.

$(A[9] \mathrm{r}-[10] \mathrm{r} ; T 8 \mathrm{v}-9 \mathrm{r})$.

La única fuente hasta ahora conocida (Corpus, 499) de esta hermosa canción era también una obra musical, esta vez vihuelística: el Libro de musica en cifras para vihuela intitulado El Parnaso... de Esteban Daza (Valladolid, 1576). Las dos versiones del poema parecen casi iguales, y sin embargo son radicalmente diferentes: no existe en la nuestra el "muerto amigo" al que llora la "niña (morenica) cuerpo garrido" en Esteban Daza. Nuestra versión no presenta el aire trágico que parece tener la otra; digo "parece", porque esa muerte del amigo podría no ser "real", sino muerte de amor. Sospecho que nuestra versión confirma el simbolismo erótico del cantar; en ella la muchacha se encuentra sola ${ }^{15}$, y sus gritos se parecen a los reclamos eróticos de la pava "en aquel monte", ante el inexperto pavón que "no la responde" (Corpus, 505), a los gritos que "dava el pastorcico en las sierras donde está" (Corpus, 502) o los que "davan en aquella sierra" llamando a Catalina o "a la más garrida" (Corpus, 191, 190 C). La escena se sitúa siempre en medio de la naturaleza: en el monte o la sierra, lugar de encuentro de los amantes y símbolo de su unión o, aquí, en un olivar, bajo un olivo, árbol igualmente simbólico, en la lírica popular española, de la unión amorosa ${ }^{16}$. En el temblar de las ramas cuaja, con una impresionante plasticidad, nada usual en esta poesía, la identificación de la morenita con el olivo; sus gritos son el temblor del árbol.

\section{Dos Gantares POPUlarizantes NO RECOGidOS EN EL CORPUS}

[9] Qué bonica eres zagala, sino que me das uida penada.

15 "Sola en l'oliuar" (con catalanismo) está en el lugar de "so el olibar" de la versión de Daza; en ésta el olivar es un olivo, un árbol, a cuya sombra se encuentra la niña; en nuestro cancionero es el campo de olivos, diferenciado del árbol (v. 5, "so el uerde oliuo"). No he podido cotejar la música de las dos versiones.

${ }^{16}$ En la lírica medieval europea "s'asseoir sous un arbre sacré -olivier, noisetier, tilleul- symbolisait une initiative que la femme pouvait prendre à l'égard d'un homme avec qui elle désirait avoir des contacts sexuels' (RIA LEMAIRE, Passions et positions. Contribution à une sémiotique du sujet dans la poésie lyrique médiévale en langues romanes, Amsterdam, 1987, p. 154). 
Eres más linda qu'el prado verde, florido y granado, más que a todo mi ganado te quiero y que a mi majada.

(A s.f.; T 33r-34r, v. 4 ganado, v. 6 te quiero a mi majda).

El estribillo ${ }^{17}$ recuerda por su estructura aquella "cantiga velha" de Camões que dice "Sois fermosa e tudo tendes, / senao que tendes os olhos verdes"' (Corpus, 112). En ninguno de los dos casos podemos saber con certeza si el estribillo es antiguo y popular; ciertamente, la glosa del cancionero, con sus dos encabalgamientos, no puede ser anterior al siglo xvi. El primer verso recuerda el "Qué bonita que es la zagala . . ', comienzo de un cantar a cuyo tono se cantaba una canción religiosa en 1609 (cf. Corpus, 2251).

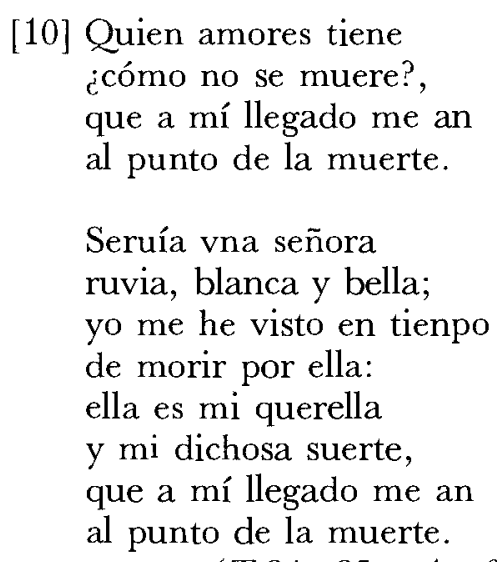

( $T 34 \mathrm{v}-35 \mathrm{v} ; A$ s.f.).

El comienzo del estribillo recuerda uno de Juan Vásquez, “Quien amores tiene, / ¿cómo duerme?..." (Corpus, 296; y cf. 718); el tema, claro está, es otro.

No sólo deben interesarnos las presencias, sino también las ausencias. Llama la atención en nuestro cancionero, junto al predominio de los villancicos, la total ausencia, por un lado, de poemas en metro italiano y, por el otro, de formas octosílabas que no sean villancicos. Se diría que, al menos en materia de poesía,

17 Sus dos versos aparecen invertidos al comienzo de la composición: " $\mathrm{Si}$ no que me das uida penada / qué bonica eres, zagala". 
los gustos del recopilador son todavía los del llamado Cancionero de Upsala, reunido en la Valencia de los años $30^{18}$. Sin embargo, también las obras polifónicas de Juan Vásquez $(1551,1560)$ muestran una análoga - aunque no tan exclusiva- preferencia por los villancicos y un gusto por la lírica popular y popularizante, lo mismo que, entre las recopilaciones poéticas de la segunda mitad del siglo, el Cancionero sevillano o los cancioneros impresos en Valencia y Barcelona (los de varios de Timoneda, la Flor de enamorados).

No hay todavía en nuestro repertorio el menor asomo del Romancero nuevo (y géneros asociados a él), que se estaba gestando en $1580^{19}$ y del cual precisamente Valencia sería uno de los grandes focos de difusión, por lo menos desde $1589^{20}$. Es ésta la razón por la cual pienso que el cancionero tuvo que haberse recopilado antes de 1582 - y quizá habría que adelantar aún más la fecha ${ }^{21}$-, en vez de situarlo vagamente en el "último tercio del siglo Xvi',

Poco a poco, con los cancioneros impresos y manuscritos que van saliendo a luz, se va configurando el panorama de los variados gustos poéticos de los españoles en aquellos tiempos. El panorama parece cambiar, no sólo de momento en momento, sino también de ciudad en ciudad y de un grupo social al otro. El cancionero musical que aquí nos ha ocupado nos permite vislumbrar lo que pueden haber sido las preferencias de los valencianos - sin duda, los de clase media- en los años inmediatamente anteriores al gran cambio de 1580 . Falta ver qué clase de música es la que contienen esos dos cuadernitos milagrosamente conservados: tarea tanto más urgente cuanto que para esos años la polifonía

18 Véase el citado artículo de Romeu Figueras, "Mateo Flecha el Viejo, la corte literariomusical del duque de Calabria..."

${ }^{19} \mathrm{Ya}$ encontramos muy desarrollado el Romancero nuevo en el manuscrito toledano 3924 de la B.N.M., del año 1582; véase Cancionero de Pedro de Rojas, pról. J. M. Blecua, eds. J. J. Labrador Herraiz, R. A. DiFranco y M. T. Cacho, Cleveland State University, Cleveland, 1988.

20 Véase Antonio Rodríguez-MoÑino, Los cancionerillos de Munich (15891602) y las series valencianas del Romancero nuevo, Estudios Bibliográficos, Madrid, 1963, pp. 9-49.

${ }^{21}$ De hecho, la pieza de Ginés Pérez pudo haber llegado a Valencia antes de que él abandonara su natal Orihuela y pasara, en 1581, a ser maestro de capilla de la catedral valenciana. En cuanto al villancico núm. 6, cuyo estribillo sólo había aparecido a partir de 1593, creo que es muy anterior a esta fecha. 
vocal en lengua vulgar cuenta con muy escasos testimonios espanoles.

\section{Índice de los villancicos}

Ai, bona moreta: nota 7

Galán que canta la misa: nota 7

Gritos daba la morenica: núm. 8

Justa cosa fue quereros [de Garci Sánchez de Badajoz]: nota 6

Mi ganado busque dueño: nota 7

Morenica, no desprecies: núm. 3

No seas cruel, señora: nota 6

(Olvida, Bras, a Constanza: nota 7)

Pux que no.m voleu amar [de Fernández de Heredia]: núm. 2

Puy fortuna m'a llevat: nota 6

Qué bonica eres, zagala: núm 9

Quien a dos amores ama: núm. 5

Quien amores tiene: núm. 10

Quién te hizo, Juan pastor: nota 7

Si el pastorcico es nueuo: núm. 7

Torna, Mingo, a enamorarte: nota 7

Váseme mi amor: núm. 4

Véante mis ojos [de Jorge de Montemayor]: núm. 1

Vente a mí, torillo: núm. 6

Zagala como las flores: nota 7

Margit Frenk

Universidad Nacional Autónoma de Méxicc 